\title{
PTENP1 is a ceRNA for PTEN: it's CRISPR clear
}

\author{
Marianna Vitiello $0^{1,2}$, Monica Evangelista ${ }^{2}$, Yang Zhang ${ }^{3}$, Leonardo Salmena ${ }^{4,5}$, Pier Paolo Pandolfi ${ }^{3,6,7^{*}}$ and \\ Laura Poliseno ${ }^{1,2^{*}}$ (D)
}

\begin{abstract}
Here we apply state-of-the-art CRISPR technologies to study the impact that PTENP1 pseudogene transcript has on the expression levels of its parental gene PTEN, and hence on the output of AKT signaling in cancer. Our data expand the repertoire of approaches that can be used to dissect competing endogenous RNA (ceRNA)-based interactions, while providing further experimental evidence in support of the very first one that we discovered.
\end{abstract}

Keywords: PTENP1, PTEN, ceRNA, CRISPR, CasRx-mediated knock-down, Cas9-mediated knock-in

\section{Main text}

In our 2010 paper entitled "A coding independent function of gene and pseudogene mRNAs regulates tumor biology", we provided the first evidence that RNA molecules, including non-coding RNAs (such as pseudogenes) and mRNAs, may be endowed with a biological function that specifically relies on their ability to compete for microRNA binding [1].

Our findings have contributed to an evolving microRNA-RNA interaction paradigm, where RNAs are not only "passive" targets of microRNAs, but also "active" regulators of microRNA availability, through a mechanism termed competing endogenous RNA (ceRNA) [2, 3]. Since our publication, a plethora of mRNAs and non-coding RNAs (lincRNAs, pseudogenes, circular RNAs) have been reported to function as ceRNAs in vitro and in animal models. Furthermore, ceRNA functions have been demonstrated to go beyond individual RNA-RNA interactions and extend into complex transcript interaction networks that can be severely dysregulated in cancer $[4,5]$.

\footnotetext{
*Correspondence: pierpaolo.pandolfiderinaldis@unito.it; laura.poliseno@cnr.it; laura.poliseno@gmail.com

${ }^{3}$ Cancer Research Institute, Beth Israel Deaconess Cancer Center, Harvard

Medical School, Boston, MA, USA

${ }^{1}$ Oncogenomics Unit, CRL-ISPRO, Pisa, Italy

Full list of author information is available at the end of the article
}

Subsequent to our 2010 publication, many studies independently confirmed PTENP1 pseudogene as a ceRNA for PTEN in prostate cancer, in other cancer types (e.g., bladder cancer, breast cancer, clear cell renal cell carcinoma, endometrial carcinoma, gastric cancer, head and neck squamous cell carcinoma, hepatocellular carcinoma), and in other physio-pathological conditions (see Supplementary references for a list). Nonetheless, a number of articles published in prestigious journals have repeatedly raised concerns about this functional interaction. Herein, we wish to address those concerns raised regarding the techniques we used to modulate PTENP1 expression and show its impact on PTEN expression.

To rule out potential non-specific effects associated with (1) supra-physiological expression of a 3'UTR [69] and (2) congestion of RNA interference machinery caused by siRNA transfection [7], we have chosen to downregulate PTENP1 expression at the transcriptional or post-transcriptional level, taking advantage of CRISPR technology.

To begin, we successfully replicated results reported in our original paper, in spite of the fact that the source of DU145 cells and the batch of siRNAs against PTEN and PTENP1 were different, and that the experiments were performed in a different lab (Fig. 1). 


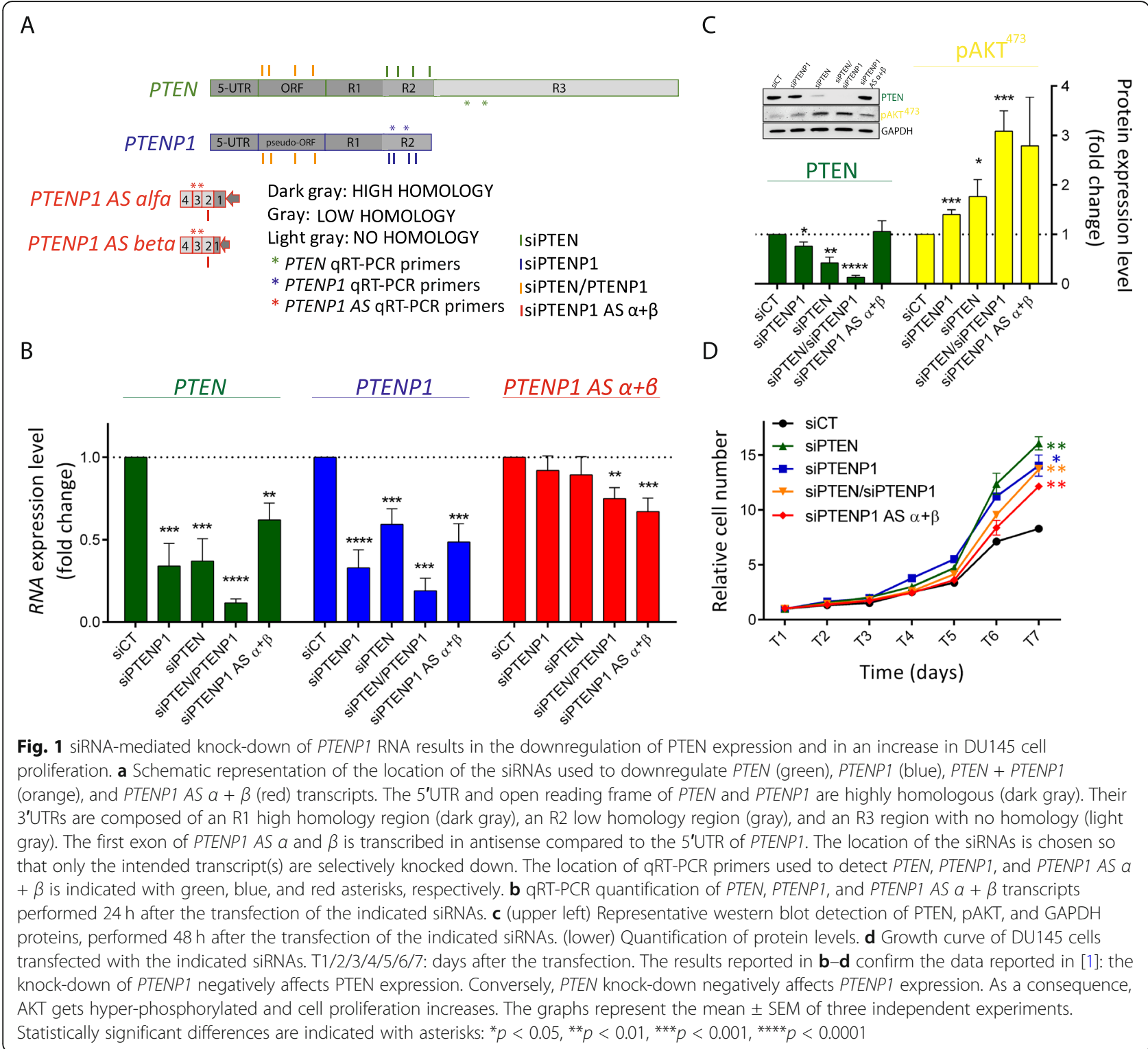

Next, in order to downregulate PTENP1 posttranscriptionally, we used the recently reported CRISPR/ CasRx system [10]. For this, we utilized 4 gRNAs designed on the same sequence of the 4 siRNAs composing the siPTENP1 mix (Fig. 2a, b) and we tested them for their ability to decrease the expression of a reporter construct in which PTENP1 3'UTR is cloned downstream of Luciferase coding sequence. As shown in Fig. 2c, the gRNAs work similarly to the corresponding siRNAs, with the mix of all 4 gRNAs working best. Therefore, we decided to use the combination of all 4 , as we had done with siRNAs. In Fig. 2d-h, we show the results obtained upon the transient transfection of the gRNA mix in GFP-sorted DU145 prostate cancer cells that stably express CasRx-eGFP (Fig. 2d, e). Consistent with the RNA interference approach (Fig. 1), the gRNA mix caused a downregulation of the intended target PTENP1 RNA, as well as of PTEN mRNA (Fig. 2f). The decrease in mRNA level was mirrored by a decrease in PTEN protein level and accompanied by increases in pAKT levels (Fig. 2g) and cell proliferation (Fig. 2h).

We also adapted the CRISPR/Cas9-based gene replacement strategy [11] in order to achieve the downregulation of PTENP1 at the transcriptional level. Specifically, we engineered an sgRNA-mediated cut between the promoter and the transcribed region of PTENP1 gene. Then, by exploiting homology-mediated recombination, we "knocked-in" a GFP expression cassette in the reverse orientation, which interferes with PTENP1 transcription (Fig. 3a). Using this strategy, we identified 11 GFP-positive KI clones (Fig. 3b), of which 7 harbored correct recombination of both homology arms 


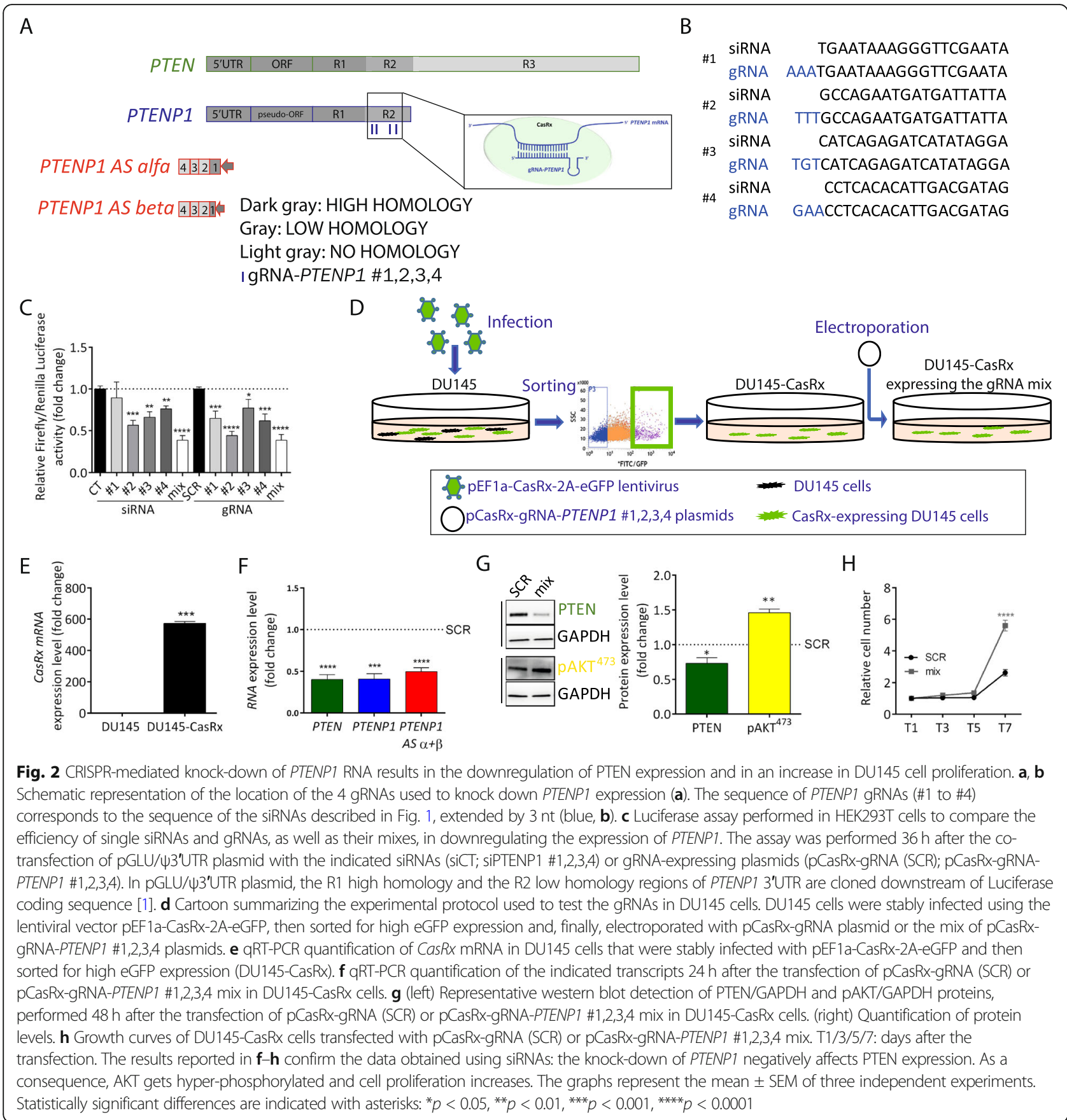

and 5 showed the expected drop in PTENP1 mRNA levels (clones \#A, 2, 5, 8, and 13 reported in Fig. 3c, d). In these clones, we also observed a decrease in both PTEN mRNA and protein levels (Fig. 3d, e). In addition, clones \#A, 2, and 13 had accompanying increases in pAKT levels (Fig. 3e) and cell proliferation (Fig. 3f). Crucially, in Fig. $3 \mathrm{~g}$, we show that endogenous PTEN mRNA levels are rescued in clone \#13, if PTENP1 3'UTR is reintroduced by means of a plasmid that expresses it downstream of Luciferase coding sequence.
In summary, using 2 CRISPR-based technologies (Figs. 2 and 3), we confirmed our results achieved using RNA interference (ref. [1] and Fig. 1): knock-down of PTENP1 leads to the repression of PTEN expression, hence the hyperactivation of oncogenic AKT signaling. In addition, we confirmed that siRNA-mediated knock-down of PTENP1 antisense alpha + beta transcripts results in a downregulation of PTENP1 and PTEN transcripts (Fig. $1 b)$, as previously reported in [12]. Conversely, we showed that the knock-down of PTEN plus PTENP1 


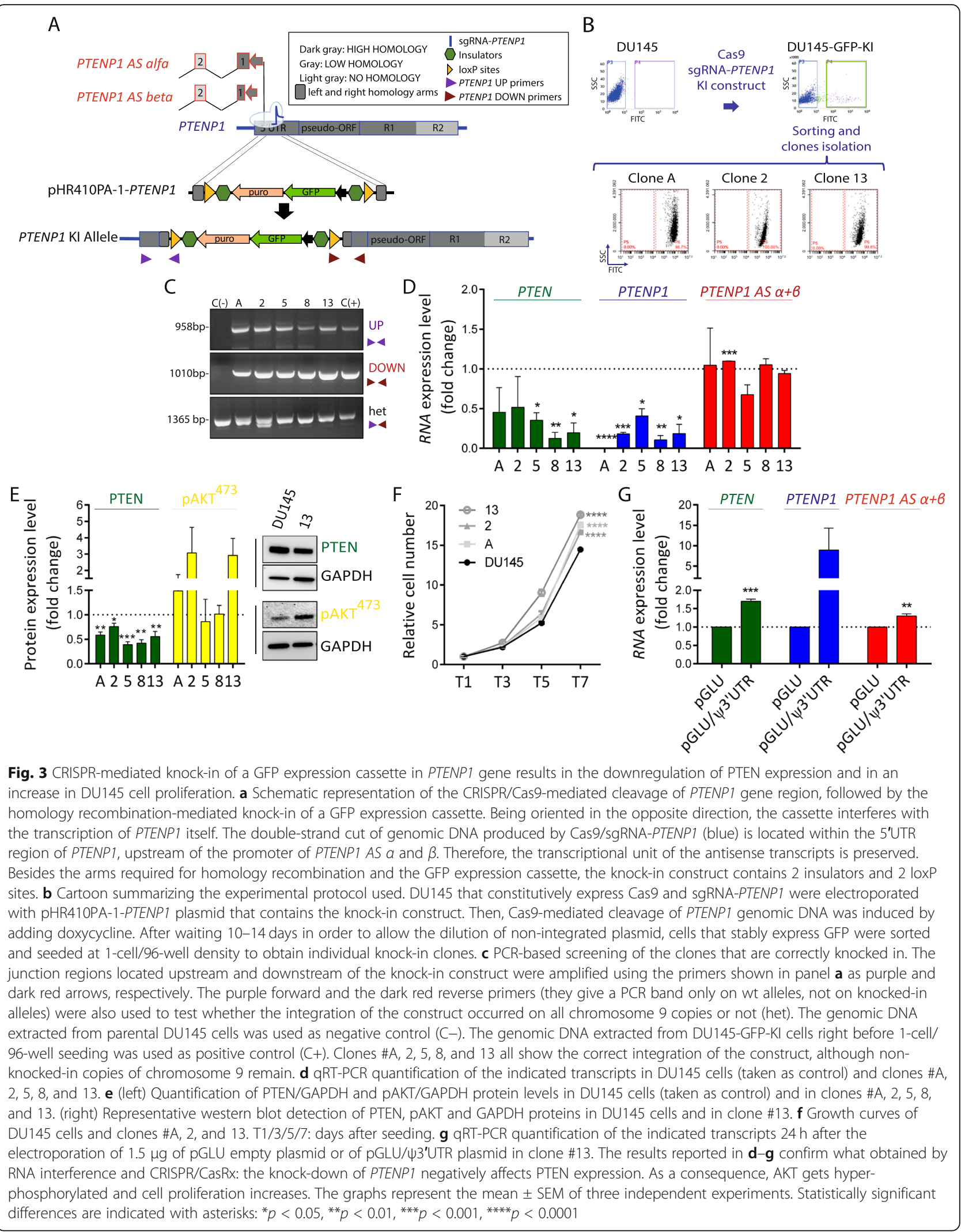


transcripts by RNA interference (Fig. 1b) and of PTENP1 by CRISPR/CasRx technology (Fig. 2f) represses the expression of PTENP1 antisense transcripts, whereas the upregulation of PTENP1 transcript elicits the opposite effect (Fig. 3g). In sum, we provide evidence that uncovers a dynamic cross-talk between PTENP1 and PTEN sense transcripts on one side and antisense PTENP1 transcripts on the other.

In the decade since our discovery, numerous groups have independently validated the regulatory interaction between PTENP1 and PTEN. Altogether, these data provide a persuasive body of work to support the existence of a robust and reproducible functional interaction between this gene-pseudogene pair [13]. Finally, the new data presented herein further reinforces the PTENP1PTEN paradigm and highlights the utility of CRISPR technologies for investigations of pseudogene-parental gene transcript relationships in cancer and other diseases.

\section{Supplementary information}

Supplementary information accompanies this paper at https://doi.org/10. 1186/s13045-020-00894-2.

Additional file 1. Supplementary references.

Additional file 2. Supplementary methods.

Additional file 3. Supplementary Figure 1. a sgRNA-PTENPI sequence. b (left) PTENP1 genomic sequence recognized by sgRNAPTENP1 (bold), and PAM sequence (5'-TGG-3', underlined). (right) Orthologous PTEN genomic sequence. sgRNA-PTENP1 cannot mediate the cleavage of PTEN because of 4 mismatches (red), one of which falls in the PAM sequence. c Electropherogram of PTENP1 genomic sequence, where the consequences of the cut by Cas9/sgRNA-PTENP1 are shown. The electropherogram was obtained by PCR analysis of the genomic DNA extracted from DU145-Cas9/sgRNA-PTENP1 double infected cells, 3 days after Cas 9 induction using $2 \mathrm{ug} / \mathrm{ml}$ doxycycline. The primers used for amplification were: Fw- attcgtcttctccccattcc; Rvtctgcaggaaatcccatagc.

\section{Abbreviations}

ceRNA: competing endogenous RNA; CRISPR: Clustered regularly interspaced short palindromic repeats

\section{Acknowledgements}

The authors thank C. Baldanzi, A. Prantera, and L. Maresca for technical support.

\section{Authors' contributions}

$M V$, PPP, and LP conceived the project. MV, YZ, and LP designed the experiments. MV and ME performed the experiments. MV, LS, PPP, and LP analyzed the data. PPP and LP supervised the research. LP wrote the manuscript with the help of all authors. The manuscript was discussed and approved by all authors.

\section{Funding}

This work was supported by ISPRO-Istituto per lo Studio, la Prevenzione e la Rete Oncologica [institutional funding to LP]. It was also partially supported by AIRC-Associazione Italiana Ricerca sul Cancro [MFAG \#17095 to LP] and by R35CA197529-01 grant to PPP. MV was supported by an AIRC fellowship.
Ethics approval and consent to participate

Not applicable

\section{Consent for publication}

Not applicable

\section{Competing interests}

None to declare

\section{Author details}

${ }^{1}$ Oncogenomics Unit, CRL-ISPRO, Pisa, Italy. ${ }^{2}$ Institute of Clinical Physiology, CNR, Pisa, Italy. ${ }^{3}$ Cancer Research Institute, Beth Israel Deaconess Cancer Center, Harvard Medical School, Boston, MA, USA. ${ }^{4}$ Department of Pharmacology and Toxicology, University of Toronto, Toronto, ON, Canada. ${ }^{5}$ Princess Margaret Cancer Centre, University Health Network, Toronto, ON, Canada. ${ }^{6} \mathrm{MBC}$, Department of Molecular Biotechnology and Health Sciences, University of Torino, Torino, Italy. ${ }^{7}$ DRI (Desert Research Institute), Renown Health, Nevada System of Higher Education, Las Vegas, NV, USA.

Received: 21 March 2020 Accepted: 5 May 2020

Published online: 09 June 2020

\section{References}

1. Poliseno L, Salmena L, Zhang J, Carver B, Haveman WJ, Pandolfi PP. A coding-independent function of gene and pseudogene mRNAs regulates tumour biology. Nature. 2010:465:1033-8.

2. Salmena L, Poliseno L, Tay Y, Kats L, Pandolfi PP. A ceRNA hypothesis: the Rosetta Stone of a hidden RNA language? Cell. 2011;146:353-8.

3. Tay Y, Rinn J, Pandolfi PP. The multilayered complexity of ceRNA crosstalk and competition. Nature. 2014;505:344-52.

4. Chan JJ, Tay Y. Noncoding RNA:RNA Regulatory networks in cancer. Int J Mol Sci. 2018;19.

5. Anastasiadou E, Jacob LS, Slack FJ. Non-coding RNA networks in cancer. Nat Rev Cancer. 2018;18:5-18.

6. Ebert MS, Sharp PA. Roles for microRNAs in conferring robustness to biological processes. Cell. 2012;149:515-24.

7. Jens M, Rajewsky N. Competition between target sites of regulators shapes post-transcriptional gene regulation. Nat Rev Genet. 2015;16:113-26.

8. Denzler R, Agarwal V, Stefano J, Bartel DP, Stoffel M. Assessing the ceRNA hypothesis with quantitative measurements of miRNA and target abundance. Mol Cell. 2014:54:766-76.

9. Broderick JA, Zamore PD. Competitive endogenous RNAs cannot alter microRNA function in vivo. Mol Cell. 2014;54:711-3.

10. Konermann S, Lotfy P, Brideau NJ, Oki J, Shokhirev MN, Hsu PD. Transcriptome engineering with RNA-targeting type VI-D CRISPR effectors. Cell. 2018;173:665-76 e614.

11. Zheng Q, Cai X, Tan MH, Schaffert S, Arnold CP, Gong X, Chen CZ, Huang S. Precise gene deletion and replacement using the CRISPR/Cas9 system in human cells. Biotechniques. 2014;57:115-24.

12. Johnsson P, Ackley A, Vidarsdottir L, Lui WO, Corcoran M, Grander D, Morris $\mathrm{KV}$. A pseudogene long-noncoding-RNA network regulates PTEN transcription and translation in human cells. Nat Struct Mol Biol. 2013;20: 440-6.

13. Kerwin J, Khan I. Reproducibility Project: Cancer B, lorns E, Tsui R, Denis A, Perfito N, Errington TM: Replication study: a coding-independent function of gene and pseudogene mRNAs regulates tumour biology. Elife. 2020;9.

\section{Publisher's Note}

Springer Nature remains neutral with regard to jurisdictional claims in published maps and institutional affiliations. 